CERN-TH.7029/93

MS-TPI-93-08

\title{
Comparison of Monte Carlo Results for the 3D Ising Interface Tension and Interface Energy with (Extrapolated) Series Expansions
}

\author{
M. Hasenbusch ${ }^{1}$ and K. Pinn ${ }^{2}$ \\ ${ }^{1}$ Theory Division, CERN, \\ CH-1211 Geneva 23, Switzerland \\ 2 Institut für Theoretische Physik I, Universität Münster, \\ Wilhelm-Klemm-Str. 9, D-48149 Münster, Germany
}

\begin{abstract}
We compare Monte Carlo results for the interface tension and interface energy of the 3-dimensional Ising model with Padé and inhomogeneous differential approximants of the low temperature series that was recently extended by Arisue to $17^{\text {th }}$ order in $u=\exp (-4 \beta)$. The series is expected to suffer from the roughening singularity at $u \approx 0.196$. The comparison with the Monte Carlo data shows that the Padé and inhomogeneous differential approximants fail to improve the truncated series result of the interface tension and the interface energy in the region around the roughening transition. The Monte Carlo data show that the specific heat displays a peak in the smooth phase. Neither the truncated series nor the Padé approximants find this peak. We also compare Monte Carlo data for the energy of the ASOS model with the corresponding low temperature series that we extended to order $u^{12}$.
\end{abstract}

CERN-TH.7029/93

MS-TPI-93-08

October 1993 


\section{Introduction}

Properties of the interface separating coexisting phases of the 3-dimensional Ising model have found continuous interest in the literature.

The Ising interface undergoes a roughening transition at an inverse temperature $\beta_{r}=0.4074(3)$ [1] that is nearly twice as large as the bulk transition coupling $\beta_{c}=0.221652(3)[2]$.

The roughening transition is believed to be of the Kosterlitz-Thouless (KT) nature [3], with the surface free energy having an essential singularity at $\beta_{r}$ of the type

$$
f_{\text {sing }} \sim \exp \left[-A\left(\beta-\beta_{r}\right)^{-1 / 2}\right] .
$$

Though the free energy and all its derivatives with respect to $\beta$ stay finite at the roughening point, one has to expect that low temperature series for interface properties suffer from the transition.

The first low temperature expansion of the 3D Ising interface tension $\sigma$ was given by Weeks et al. to $9^{\text {th }}$ order in the variable $u=\exp (-4 \beta)$. Shaw and Fisher [4] analyzed the series with the help of Padé and inhomogeneous differential approximants. They claimed that the Padé and differential approximants allow to compute the surface tension accurately for temperatures below the roughening point.

Recently, Arisue put forward the series to $17^{\text {th }}$ order in $u$ [5]. It is interesting to note that the coefficients of the series change their sign at order 13. This behavior does not come unexpected and confirms that the roughening transition of the Ising interface is of Kosterlitz-Thouless type: Expanding eq. (1) in the variable $u$, one also obtains a series with coefficients that change their sign at a certain order. The order where the change of sign happens depends on the non-universal parameters $A$ and $\beta_{r}$.

A completely independent method to study the Ising interface is the Monte Carlo method [6]. For recent Monte Carlo work on the 3D Ising interface see, e.g., [1, 8, 9, 10, 11, 12] and references cited therein.

In [9], we reported on a numerical study of properties of the Ising interface over the whole range from low temperatures up to the bulk critical point. In particular we determined the surface energy and, by integration over $\beta$, also the surface free energy and surface tension.

In this paper, we give a more detailed account of the numerical results and compare them with Padé and inhomogeneous differential approximants for the extended low temperature series. In order to demonstrate that the disagreements of series and numerical data are not due to finite size effects, we provide data for various lattice extensions and demonstrate that the systematic errors in the determination of surface energy and surface tension are under control.

The ASOS (absolute value solid-on-solid) model in two dimensions is an approximation of a phase separating surface in a 3-dimensional Ising model. The approximation neglects overhangs of the surface and fluctuations of the bulk phases. For temperatures being low compared to the bulk critical temperature of the Ising model this approximation should describe the Ising surface quantitatively rather well. We extended the existing low temperature series for the ASOS model to $12^{\text {th }}$ order and 
compared its Padé approximants with Monte Carlo results that we obtained with the VMR cluster algorithm [13].

This paper is organized as follows. In section 2 we introduce the notation for the 3D Ising model and its ASOS approximation. We also define the interface tension and discuss the problem of how to obtain it from simulations of finite lattices. The algorithms used for the Monte Carlo simulations are specified in section 3. In section \$ we quote the results and compare them with extrapolations of low temperature series. Conclusions are given in section 5 .

\section{The Models and Quantities Studied}

\section{3-Dimensional Ising Model}

We consider a simple cubic lattice with extension $L$ in $x$ - and $y$-direction and with extension $t$ in $z$-direction. The Ising model is defined by the partition function

$$
Z=\sum_{\left\{\sigma_{i}= \pm 1\right\}} \exp (-\beta H)
$$

The Hamiltonian $H$ is a sum over nearest neighbor contributions,

$$
H=-\sum_{<i, j>} k_{i j} \sigma_{i} \sigma_{j}
$$

The interaction energy is normalized such that $\beta=J / k_{\mathrm{B}} \tilde{T}$, where $k_{\mathrm{B}}$ denotes Boltzmann's constant, $J$ is the interaction energy, and $\tilde{T}$ is the temperature.

The lattice becomes a torus by defining that the uppermost plane is regarded as the lower neighbor plane of the lower-most plane. An analogous identification is done for the other two lattice directions. For the Ising spins $\sigma$ we use two different boundary conditions: Periodic boundary conditions are defined by letting $k_{i j}=1$ for all links $\langle i, j\rangle$ in the lattice. To define antiperiodic boundary conditions in $z$-direction, we also set $k_{i j}=1$ with the exception of the links connecting the uppermost plane with the lower-most plane. For these links we set $k_{i j}=-1$.

Let us define

$$
G=-\left(\ln Z_{a}-\ln Z_{p}\right)
$$

where the subscript $a(p)$ means antiperiodic (periodic) boundary conditions. The surface tension can be defined as the limit

$$
\sigma=\lim _{t \rightarrow \infty} \lim _{L \rightarrow \infty} \frac{G}{L^{2}}
$$

With numerical simulations only a rather limited range of $L$ and $t$ values is accessible. Hence a careful discussion of finite size effects is needed. Let us express the partition functions of the periodic and antiperiodic Ising system in terms of the transfer matrix $T$. The antiperiodic boundary conditions are represented by a spin-flip operator $P$ that flips the sign of all spins in a given $z$-slice. 
The partition function of the periodic system is given by $Z_{p}=\operatorname{Tr} T^{t}$, while the partition function of the antiperiodic system is given by $Z_{a}=\operatorname{Tr} T^{t} P$. The operators $T$ and $P$ commute and thus have a common set of eigenfunctions. Say the eigenvalues of $T$ are $\lambda_{i}$ and those of $P$ are $p_{i}$. The possible values of $p_{i}$ are 1 and -1 . The partition functions take the form $Z_{p}=\sum_{i} \lambda_{i}^{t}$ and $Z_{a}=\sum_{i} \lambda_{i}^{t} p_{i}$.

Let us consider the ratio of the partition functions in the broken phase of the model. Here the two largest eigenvalues $\lambda_{0 s}$ and $\lambda_{0 a}$ are much larger than the other eigenvalues [14]. (The subscripts $s$ and $a$ label eigenfunctions with $p=1$ and $p=-1$, respectively.) Hence the ratio of the two partition functions can be well approximated by

$$
\frac{Z_{a}}{Z_{p}} \simeq \frac{\lambda_{0 s}^{t}-\lambda_{0 a}^{t}}{\lambda_{0 s}^{t}+\lambda_{0 a}^{t}} .
$$

The corrections are of order $\left(\lambda_{1 s} / \lambda_{0 s}\right)^{t}$. This means that the extension $t$ of the lattice in $z$-direction has to be much larger than the bulk correlation length $\xi$. For $\xi_{0 a}>>t$ $\left(\xi_{0 a}=-1 / \ln \left(\frac{\lambda_{0 a}}{\lambda_{0 s}}\right)\right.$ is the tunneling correlation length) we can write

$$
\frac{Z_{a}}{Z_{p}} \simeq \frac{t}{2}\left(1-\frac{\lambda_{0 a}}{\lambda_{0 s}}\right) .
$$

Notice that within this approximation the derivative of $G$ with respect to $\beta$ does not depend on $t$.

According to this discussion, if $\xi<<t<<\xi_{0 a}$ then already for finite $L$ a surface free energy is rather well defined by

$$
F_{s} \simeq G+\ln t
$$

Phenomenologically one can interpret this situation as follows:

The lattice is short enough that the creation of interfaces in the system with periodic boundary conditions is sufficiently suppressed while for the system with antiperiodic boundaries only the interface induced by the boundary conditions is present. On the other hand the extension of the lattice is large enough not to restrict the fluctuations of the interface.

In order to discuss the $L$ dependence of the surface free energy a model for the surface is needed.

Theoretical studies of the interface are based on the SOS (solid-on-solid) approximation which essentially neglects overhangs and bulk fluctuations. SOS models predict that the roughening transition is of the Kosterlitz-Thouless type [15]. That the Ising interface at the roughening point is indeed in the same universality class as various SOS models was demonstrated by Hasenbusch using a renormalization group matching procedure [1]. For large $\beta$ there are also rigorous results from linked cluster expansions. Borgs and Imbrie have shown [16] for sufficiently large $\beta$, i.e., when the interface is smooth, that

$$
F_{s} \simeq \sigma L^{2}
$$

It is believed that this result holds for all $\beta>\beta_{r}$. 
A model for the interface in the rough phase is the capillary wave model [17]. In its quadratic approximation it essentially states that the infrared fluctuations of the interface are massless Gaussian. This assumption has been verified numerically in a number of cases, see e.g. [9]. The massless Gaussian dynamics leads to the following finite $L$ behavior of the surface free energy in the rough phase [18, 19, 20, 21]:

$$
F_{s} \simeq C_{s}+\sigma L^{2}
$$

Gelfand and Fisher 22] predicted in addition a logarithmic dependence of the surface free energy on the lattice size. They did not take into account a prefactor $L$ in the partition function that arises when the average position of the interface is fixed via a $\delta$-function.

At the roughening transition one has still a Gaussian fixed point, however, with logarithmic corrections. Hence eq. (10) should be still valid for sufficiently large $L$.

It is difficult to compute free energies directly by Monte Carlo (however, cf. [11]). But the derivative of $G$ with respect to $\beta$ is a quantity that can be computed by Monte Carlo:

$$
\frac{\partial G}{\partial \beta}=\langle H\rangle_{a}-\langle H\rangle_{p} \equiv E_{s}
$$

$G$ can then be obtained by integration over $\beta$ :

$$
G(\beta)=G\left(\beta_{0}\right)+\int_{\beta_{0}}^{\beta} d \beta^{\prime} E_{s}\left(\beta^{\prime}\right),
$$

where $\beta_{0}$ is arbitrary. In the case that there is only one interface in the system, $E_{s}$ is the surface energy. The surface energy per area is defined as

$$
\epsilon_{s}=E_{s} / L^{2}
$$

In 19] we used the method of 'integration over $\beta$ ' to determine the surface free energies for a wide range of temperatures, for $L=8,16,32,64$. We found that the surface free energy can be fitted accurately with eq. (10). We thus identify the coefficient $\sigma$ in front of the factor $L^{2}$ with the surface tension.

\section{ASOS Model}

The ASOS model is the solid-on-solid approximation of an interface of a 3D simple cubic lattice Ising model (on a (001)-lattice plane). It lives on a two-dimensional square lattice of size $L$ by $L$. The partition function is

$$
Z_{A S O S}=\sum_{h} \exp \left(-\beta \sum_{<i j>}\left|h_{i}-h_{j}\right|\right),
$$

with $h_{i}$ integer. We shall study the quantity

$$
\epsilon_{A S O S} \equiv E_{A S O S} / L^{2}=<\sum_{<i, j>}\left|h_{i}-h_{j}\right|>/ L^{2}
$$


In the ASOS limit of the Ising model the inverse temperatures of the two models are related by

$$
\beta_{\text {Ising }}=\beta_{A S O S} / 2 .
$$

In the same limit, there is the following relation between the surface energies:

$$
E_{s, \text { Ising }}=2+2 E_{A S O S}
$$

for corresponding $\beta$ values. The most recent estimate for the roughening coupling of the ASOS model is $\beta_{r, A S O S}=0.8061(3)$ [23].

\section{Monte Carlo Algorithms}

\section{Ising Model}

\section{Cluster Algorithm for the Ising Interface}

For the production of the Monte Carlo results of ref. [9] and for part of the new results to be presented below we employed the Ising interface cluster algorithm of Hasenbusch and Meyer [24]. A detailed description of this algorithm can be found in [9]. There we computed besides the surface energies also nonlocal quantities like the surface thickness and block spin correlation functions. The cluster algorithm proved to be very efficient for this purpose.

\section{Local Demon Algorithm for the Ising Model}

When the focus is on the determination of the energy with antiperiodic boundary condition, it is helpful to use a local algorithm instead of the cluster algorithm. It is much easier to adapt a local Monte Carlo algorithm for vectorization, parallelization or multi-spin coding.

It turned out that the energy of the system with antiperiodic boundary conditions does almost not couple to the slow modes of the local algorithm.

For the update of the 3-dimensional Ising model we therefore also used a microcanonical demon algorithm [25, 26, 27] in combination with a particularly efficient canonical update [28] of the demons. The algorithm is implemented using the multispin coding technique [26, 27]. Every bit of a computer word carries one Ising spin. In order to avoid restrictions of the geometry of the systems 32 independent systems are run in parallel. The demon carries three levels carrying the energies 4, 8 and 16 . The number of demons is chosen to be equal to the number of lattice sites.

The simulation is done by performing a cycle of 5 groups, where each group consists of a microcanonical update of the spin demon system and a translation of the demon layer with energy 4,8, or 16 (alternating). Each group is finished by

updating the demons with energy 4 . The hole cycle is completed by updating the demons with energy 8.

The canonical update of a demon layer consists of the following steps [28]:

1. calculate the total number $N_{\text {old }}$ of demons in that layer carrying energy 
2. calculate a new total number $N_{\text {new }}$ of demons with energy according to the probability $p_{E}=n_{E} \exp (-\beta E) / Z_{D}$, with $Z_{D}=\sum_{E} n_{E} \exp (-\beta E)$, where $n_{E}$ is the number of demon states having the total energy $E$

3. if $N_{\text {new }}>N_{\text {old }}$ then pick randomly $N_{\text {new }}-N_{\text {old }}$ empty demons and fill them, else pick randomly $N_{\text {old }}-N_{\text {new }}$ occupied demons and clear them

This is a valid update of the total demon system that provides new total energies with a heat bath distribution. Random numbers are only needed for the selection of the $\left|N_{\text {old }}-N_{\text {new }}\right|$ demons which change their energy and one for the selection of the new total energy. Notice that $\left|N_{\text {old }}-N_{\text {new }}\right|$ is only of the order of the square root of all the demons.

Combined with shifts and translations of the demons such an update of the demons should be almost as good as a heat bath for every single demon.

In table 11 we quote the performance of our algorithm on various workstations. For comparison we also cite the performance data of algorithms of other authors on different supercomputers in table 2 .

\section{VMR Algorithm for the ASOS Model}

The results for the energy of the ASOS model cited in this paper were obtained with the help of the VMR (valleys-to-mountains-reflection) algorithm introduced in [13]. The basic idea of this algorithm is to define valleys and mountains by cutting the configuration of height variables with a reflection plane. The flip of a cluster of spins can be regarded as reflecting a valley to a mountain (or vice versa). This type of algorithm has proved very successful for various SOS models.

\section{Discussion of Results}

\section{Ising Model}

\section{The Surface Energy}

We first explain how we obtained estimates for the surface energy defined in eq. (11). The quantity $\langle H\rangle_{a}$ was always computed using either the interface cluster algorithm or the local demon algorithm. For the determination of the energy with periodic boundary conditions we used a Padé approximant of the low temperature series for the energy (for details cf. [9]). The series was put to order 24 in $u=\exp (-4 \beta)$ by Bhanot et al. [29] and a little later to order 32 by Vohwinkel [30]. In [9], we found by comparing with Monte Carlo results, that the use of the Padé approximation was safe for $\beta \geq 0.26$ for $L=8, \beta \geq 0.24$ for $L=16$ and $L=32$, and for $\beta \geq 0.235$ for $L=64$. For the range of couplings that we want to focus on in this paper $(\beta \geq 0.35)$, the use of the Padé approximant is safe (for the accuracy required). 
When computing the surface energy per area $\epsilon_{s}$ one has to deal with systematic effects from the finite extension of the lattice in $t$ - and in $L$-direction.

First we carefully studied the $t$-dependence of the surface energy for three different $\beta$-values, namely $\beta=0.45$ (which is in the smooth phase of the interface), $\beta=0.4074$ (the roughening point) and $\beta=0.3500$ (which is in the rough phase of the interface, but still far away from the bulk critical point). The results for $\epsilon_{s}$ for interfaces with extension $L=4,8,16,32,64$ and for lattices with various values of $t$ are quoted in the tables 3 , 4 and 5. The statistics is as follows: For the runs with the cluster algorithm we performed 400000 measurements, separated by an update step made up from generating and flipping 8 clusters of two different types, cf. [9], and a subsequent Metropolis sweep. For the runs with the demon algorithm we made 100000 measurements on each of the 32 independent copies, separated by always 5 cycles as described in section 3. For some sets of parameters we made runs with two different random number generators and found perfect consistency. As a further check we compared Monte Carlo results for $L=t=4$ with the exact result for the energy.

An inspection of the tables reveals that (for fixed $L$ ) the estimates for $\epsilon_{s}$ are consistent within error bars for $t \geq 4$ and $\beta=0.45$. For $\beta=0.4074$ we find consistency for $t \geq 5$ if $L \leq 32$ and for $t \geq 6$ if $L=64$, cf. the discussion of finite $t$ effects in section 2. In this context it might be interesting to note that the bulk correlation length $\xi$ which governs the exponential decay of the connected correlation function is 0.2809 for $\beta=0.45$. For $\beta=0.4074$, one has $\xi=0.3162$, and for $\beta=0.35$ the correlation length is $\xi=0.3897$. These estimates are based on a Padé evaluation of a low temperature series by Arisue and Fujiwara [31]. Correlation length estimates from the series truncated at $13^{\text {th }}$ order for $\beta=0.35$ differ from the Padé approximants in the third digit.

Using the safe values of $t$ found for the smaller $\beta$-values we computed the interface energies presented in table 6 for the range from $\beta=0.35$ to $\beta=0.6$ in steps of 0.01 or 0.005 . The table also contains estimates for the $L=\infty$ interface energy from the low temperature series, truncated at $17^{\text {th }}$ and at $12^{\text {th }}$ order, cf. the discussion below.

Concerning the convergence of the surface energies to the infinite $L$ limits we make the following observations: For $\beta \geq 0.45$ we find that (within the statistical accuracy obtained) the estimates converged well, consistent with exponentially small $L$-corrections. Of course, the convergence becomes better for larger $\beta$.

In the rough phase the interface energy is expected to behave like $A+B L^{2}$. We fitted the $\beta=0.35$ data for $L \geq 8$ and $t=13$ with this ansatz and found $A=-5.96(47)$ and $B=3.70428(9)$ with $\chi^{2} /$ dof $=1.135$. 'f

At the roughening transition the situation is more difficult. We studied the differences $E_{s}(2 L)-E_{s}(L)$ for $L \leq 64$ and found that this quantity scales down with a factor of at least 3 always when $L$ is doubled. This gives us confidence that $E_{s}(\infty)-E_{s}(128)$ is not bigger than $E_{s}(128)-E_{s}(64)=0.0022(1)$.

\footnotetext{
${ }^{1}$ For a study of finite size effects in a previous high precision simulation performed by Ito see 12

${ }^{2}$ Infinite $L$-extrapolations according to the law $A+B L^{2}$ for data in the rough phase were also done by Ito 12
} 
The estimates for the interface energies can be compared with results from the low temperature expansion of the same quantity. The series for the interface tension is

$$
\sigma=2 \beta+\sum_{n=2}^{17} a_{n} u^{n}+\mathcal{O}\left(u^{18}\right) .
$$

The coefficients up to order $u^{9}$ in the low temperature variable $u=\exp \left(-4 \beta_{\text {Ising }}\right)$ were first determined by Weeks et al. They can be found in a paper by Shaw and Fisher [四]. The higher coefficients (order $u^{10}$ through $u^{17}$ ) were computed recently by Arisue [5]. The coefficients $a_{n}$ are quoted in table 9. In order to get $\epsilon_{s}$ from $\sigma$, one has to differentiate eq. (18) with respect to $\beta$. The result is a power series in $u$ (there is no longer a term $\ln (u)$ present). We compared the truncated series and its Padé approximants with the Monte Carlo results as follows: For order $k$, with $7 \leq k \leq 17$, we plotted the result of the truncated series (truncated at order $k$ ) together with the results of 4 or 5 close-to-diagonal Padé approximants $[m / n]$, with $m+n=k$. For even $k$ we took the five Padeś with $m=k / 2-2, \ldots, k / 2+2$, for odd $k$ we took the four Padés with $m=(k-1) / 2-1, \ldots,(k-1) / 2+2$. In all figures the truncated series estimates are plotted with a ' + ' and connected with a broken line. For the Padé estimates we use diamonds. On the right hand side of the plots we present our Monte Carlo estimates of the surface energy for different lattice sizes. The $L=64$ result is also plotted with two vertical lines for easier comparison with the series results.

Figure 1 shows this comparison for $\beta=0.50$ which is in the smooth phase of the interface and far away from the roughening transition. For order $\geq 16$ the truncated series and the Padé approximants have become consistent with the Monte Carlo estimate. Note however, that there is no apparent advantage in using the Padé approximants instead of the truncated series.

$\beta=0.45$ is still in the smooth phase. The comparison is summarized in figure 2. Notice the much larger scale of the $y$-axis in this plot compared to figure 1 . The Padé approximants scatter a lot, especially around order 13 where the series changes its sign. If one looks only at order $\leq 12$, the truncated series seems to be even superior to the Padé approximations.

The scenario becomes even more drastic if one proceeds to the roughening region. In figure 3 we show the comparison of the different approximations at the roughening point $\beta=0.4074$. Here obviously neither the truncated series nor the Padé approximants lead to a reasonable approximation.

\section{The Surface Tension}

The estimates for the surface tension quoted in this paper were obtained with the method described in [9]. [ ]

However, for the $\beta$-range above 0.35 we used the new and much more precise estimates for the surface energy as quoted in table 6. From the results for the

\footnotetext{
${ }^{3}$ We plan to give a comparison of the different methods to determine the interface tension (see, e.g., [7, 8, 9, 10, 11, 12]) in a forthcoming publication [32]
} 
surface energy we determined the surface tension using the method of 'integration over $\beta^{\prime}$ as outlined in section 2. In [9] we used two different starting points $\beta_{0}$ for the integration, namely $\beta_{0} \approx \beta_{c}$ and $\beta_{0}=0.6$. Both methods yielded compatible results. In table 7 we quote our new results obtained by starting the integration at the following $\beta$ values: For $L=8$ and $L=16$ we took $\beta_{0}=0.545$, for $L=32$ we used $\beta_{0}=0.515$, and for $L=64$ we started the integration at $\beta_{0}=0.495$. The starting point was determined such that the Monte Carlo surface energy estimate and the $17^{\text {th }}$ order low temperature series for the same quantity are consistent within the present error bars. Note that the errors quoted in table 7 are statistical errors ( $1 \sigma$ error bars) that do not include systematic effects. The estimates rely on fits of the finite $L$ behavior of the free energy with the law $C_{s}+\sigma L^{2}$.

For the estimation of systematical errors we used the following procedure: One defines

$$
\sigma(L)=F_{s}(L) / L^{2}
$$

By the very definition this quantity converges to the interface tension in the infinite $L$ limit, however, with stronger finite size effects than definition eq. (10). So looking at the variation of $\sigma(L)$ gives one a feeling of the maximal systematic error possible.

It is also instructive to obtain estimates for $\sigma$ based on the law eq. (10), however, using just pairs of adjacent $L$-values. Then no fit is needed. In table 8 we quote these quantities for $\beta=0.402359$ (which corresponds to $u=0.2$ ) and for $\beta=0.45$. We adopt the following rule for the estimation of a systematical error: Take the estimates for $\sigma$ from the pair $L=16,32$ and from the pair $L=32,64$ and compute the difference. Take this as the systematic error of the interface tension determined with the fit method. For the two examples studied in table 8 we conclude that for $\beta=0.45$ the systematic error is smaller than the statistical error. For $\beta=0.402359$ we arrive at $\sigma /(2 \beta)=0.84487(3)$.

In figures 4 and 5 we show the comparison of the Monte Carlo results for the two $\beta$ values quoted abovef with the truncated series and the Padé approximations. The Padés were not performed directly for the series for $\sigma$ but (as in [4]) for the quantity $Q(u)=u \exp (2 \sigma)$.

The conclusions are similar to the ones for the surface energy. Figure 5 demonstrates the trap one can get into when the series is too short. Shaw and Fisher might have been misled by the convergence and consistency of the Padé and differential approximants at order 9 and concluded that the interface tension could well be approximated by Padés of ninth order for temperatures below the roughening temperature. The now longer series shows that this is a wrong conclusion. Note that the Padés seem to converge again at the by now highest available order. But still, the value is definitely off from our Monte Carlo estimate.f

\footnotetext{
${ }^{4}$ We chose $u=0.2(\beta=0.402359)$ for easier comparison with the work of Shaw and Fisher. We compared our Monte Carlo results with the series extrapolations also at the more recent estimate $\beta_{r}=0.4074$, with the same conclusions

${ }^{5} \mathrm{~A}$ preliminary Padé analysis for the interface tension was already performed by Arisue in [5]. His results are perfectly consistent with ours. However, he could not compare with an independent Monte Carlo result
} 
Shaw and Fisher pointed out that the use of inhomogeneous differential approximants [33] might be superior to using Padés. In fact, these approximants generalize Padés and are suitable to deal with functions that have a critical behavior like $\sim A(x)\left(x-x_{c}\right)^{-\gamma}+B(x)$. Note, however, that the singularity of the free energy of the Ising interface is not of this type.

Table 10 shows the results obtained by evaluating inhomogeneous differential approximants for $\sigma /(2 \beta)$ at $\beta=0.402359$ which corresponds to $u=0.2$. Like Shaw and Fisher, we computed the approximant for the quantity $Q(u)$ defined above and then took the logarithm. We here only discuss the order 9 and order 17 approximants. The order 9 approximants are fairly stable, however yield too small results (as the Padé approximants of this order do). Recall that the Monte Carlo estimate for this $\beta$-value is $\sigma /(2 \beta)=0.84487(3)$. The order 17 approximants are also fairly stable, however, they now overshoot the Monte Carlo estimate definitely. We conclude that using inhomogeneous differential approximants does not cure the problem (as was to be expected). The analysis of the surface tension at $\beta=0.4074$ leads to a similar result.

In fig. 6 we compare our results for the surface tension with the whole range approximant of Fisher et al. [34, 4, 35] in the $\beta$-range $0.35-0.50$. The approximant provided by Fisher and Wen [34] is obtained as discussed in ref. [4]. In addition the critical amplitude of the surface tension is fixed to the value given by Mon [36]. The mismatch of the two curves can be explained by the failure of the approximants to the low temperature series of order 9 at $u=0.20$. The surface tension at $u=0.20$ is underestimated and since the surface energy is overestimated the gap between the two curves increases with decreasing $\beta$.

For $\beta$-values close to the bulk critical temperature the Monte Carlo result and the interpolation are consistent again. This fact confirms the validity of the result for the surface tension amplitude obtained by Mon [36].

\section{The Specific Heat}

The specific heat of models undergoing a KT phase transition was investigated in a number of Monte Carlo studies. For the XY model a peak of the specific heat is found, which is located in the massive phase of the model at about $0.9 \beta_{c}$, see e.g. refs. [37, 38, 39]. Swendsen [40] also found a peak of the specific heat for the DGSOS and ASOS models in the massive (smooth) phase of the models. For the BCSOS model (or F-model) that is an SOS model with the constraint that two neighboring height variables must differ by +1 or -1 , the specific heat can be computed exactly (chapter 8 in the book of Baxter [11). The peak of the specific heat lies clearly in the massive phase.

In contrast to these findings, Shaw and Fisher [4] arrive at the conclusion (based on their series analysis) that the peak of the specific heat of the 3D Ising interface is located in the massless (rough) phase.

The position of the specific heat peak is, of course, not a universal feature of the KT transition since the specific heat stays finite for all temperatures. However, the ASOS model is supposed to approximate the Ising interface at the roughening 
transition even quantitatively quite well. Hence it would be quite surprising if the specific heat peak of the Ising interface should be in the massless phase while that of the ASOS model is located in the massive phase.

We computed the derivative of the surface energy with respect to the inverse temperature $\beta$ from finite differences of the energy. The results for $L=8,16,32$ and 64 are given in fig. 7. For comparison we give the truncated series result for order 12 and 17. For the lattices of size $L=16,32$ and 64 the derivative of the energy clearly

exhibits a peak for $\beta \sim 0.43>\beta_{r}$. The peak of the specific heat $C=-\frac{1}{T^{2}} \frac{d E}{d \beta}$ itself is even slightly deeper in the massless phase. This is in contradiction to the Shaw and Fisher result. The peak height still increases considerable for $L=64$ compared to $L=32$. This fact indicates that length scales of order 10 lattice spacings are largely involved in the generation of the specific heat peak. On the other hand the correlation length at $\beta=0.43$ is finite. It should be of order 100 .

For $\beta>0.46$ the derivative of the energy obtained from the $12^{\text {th }}$ and $17^{\text {th }}$ order truncated series are close together and reproduce the Monte Carlo result within error bars. The $12^{t h}$ order truncated series contains only coefficients with positive sign and is hence increasing monotonically with increasing $u$. Obviously it cannot predict the peak of the specific heat. The situation is slightly different for the $17^{\text {th }}$ order truncated series. The curve displays a peak at $\beta=0.3747 \ldots$ deep in the massless phase, but obviously wrong.

One expects the low temperature series of the free energy to converge for $\beta>\beta_{r}$. Hence in principle one should be able to obtain the specific heat peak accurately from the truncated series of sufficiently high order. However, since length scales of more than ten are involved one would have to compute diagrams of this extension.

\section{ASOS Model}

It is instructive to study the same questions in the SOS approximation. The motivation for this is twofold: First, the comparison of the results demonstrates that the problem studied is indeed due to the Kosterlitz-Thouless nature of the roughening transition. Furthermore, e.g. in the ASOS model it is much easier to get precise data for large $L$. Table 11 shows our Monte Carlo results for the energy of the ASOS model as introduced in section 2. The data were obtained with the help of the VMR algorithm. Every single simulation consisted of at least 20000 updates.

In table 9 we quote the coefficients of the low temperature expansion of the free energy per area for the ASOS model. The expansion variable is $u=\exp \left(-4 \beta_{\text {Ising }}\right)$, with $\beta_{\text {Ising }}=\beta_{\mathrm{ASOS}} / 2$. The series is

$$
\sigma_{\mathrm{ASOS}}=2 \beta+\sum_{n=2}^{12} a_{n} u^{n}+\mathcal{O}\left(u^{13}\right) .
$$

The coefficients $a_{n}$ with $n \leq 9$ are due to Weeks et al. (cited in [4]). We extended this series to order 12, using a technique due to Arisue and Fujiwara [31, 5]. Our result for the ninth order is at odds with the result cited in [4], where $a_{9}=3185 / 3$ is quoted. We used our own value in what follows. 
It is interesting to note that the series (like the corresponding Ising series) has a characteristic change of sign in the coefficients. From the series for the free energy one easily obtains the series for the energy per area. Figures 8 and 9 compare again our Monte Carlo results with the truncated series and with Padé approximants of increasing order. The observations are essentially the same as for the Ising model. Since we extended the series beyond the order where the change of sign takes place we can observe the Padé approximants becoming unstable around this order.

\section{Conclusions}

In this paper, we presented a comparison of Monte Carlo results for interface properties with low temperature series. We took the obvious discrepancy between the methods as a motivation to improve confidence in the Monte Carlo estimates by providing a detailed study of possible systematic errors.

The failure of the series approximations to improve the series result compared with the truncated series as discussed in this article is not completely unexpected. However, our detailed study reveals the seriousness of the problem and calls for new approaches to deal with essential singularities in series expansions.

\section{Acknowledgment}

We would like to thank M.E. Fisher for detailed and stimulating correspondence about the subjects discussed in this article. We thank H. Arisue for communicating his series results prior to publication. We thank M.E. Fisher and H. Wen for sending us detailed data of their interface tension estimates.

\section{References}

[1] M. Hasenbusch, PhD thesis, Universität Kaiserslautern, 1992.

[2] C.F. Baillie, R. Gupta, K.A. Hawick and G.S. Pawley, Phys. Rev. B 45 (1992) 10438.

[3] J.M. Kosterlitz and D.J. Thouless, J. Phys. C 6 (1973) 1181;

J.M. Kosterlitz, J. Phys. C 7 (1974) 1046.

[4] L.J. Shaw and M.E. Fisher, Phys. Rev. A 39 (1989) 2189.

[5] H. Arisue, preprint OPCT 93-1, May 1993.

[6] K. Binder (ed.), Monte Carlo Methods in Statistical Physics, Springer, Berlin 1986.

[7] S. Klessinger, G. Münster, Nucl. Phys. B 386 (1992) 701.

[8] B.A. Berg, U. Hansmann and T. Neuhaus, Z. Phys. B 90 (1993) 229. 
[9] M. Hasenbusch and K. Pinn, Physica A 192 (1993) 342.

[10] H. Gausterer, J. Potvin, C. Rebbi and S. Sanielevici, Physica A 192 (1993) 525.

[11] M. Hasenbusch, J. Phys. I France 3 (1993) 753.

[12] N. Ito, Physica A 196 (1993) 591.

[13] H.G. Evertz, M. Marcu, K. Pinn and S. Solomon, Phys. Lett. B 254 (1991) 185.

[14] V. Privman and M.E. Fisher, J. Stat. Phys. 33 (1983) 385.

[15] S.T. Chui and J.D. Weeks, Phys. Rev. B 14 (1976) 4978;

T. Ohta and K. Kawasaki, Prog. Theor. Phys. 60 (1978) 365;

For reviews on SOS models see, e.g.:

D.B. Abraham, Surface Structures and Phase Transitions - Exact Results, in Phase Transitions and Critical Phenomena Vol. 10, C. Domb and J.L. Lebowitz (eds.), Academic, 1986, p. 1;

H. van Beijeren and I. Nolden, The Roughening Transition, in Topics in Current Physics, Vol. 43: Structure and Dynamics of Surfaces II, W. Schommers and P. von Blanckenhagen (eds.), Springer, 1987, p. 259;

J. Adler, Physica A 168 (1990) 646.

[16] C. Borgs and J.Z. Imbrie, Comm. Math. Phys. 145 (1992) 235

[17] V. Privman, Int. J. Mod. Phys. C 3 (1992) 857.

[18] E. Brézin and J. Zinn-Justin, Nucl. Phys. B 257 (1985) 867.

[19] G. Münster, Nucl. Phys. B 340 (1990) 559.

[20] B. Bunk, Int. J. Mod. Phys. C 3 (1992) 889.

[21] M. Caselle, F. Gliozzi and S. Vinti, Phys. Lett. B 302 (1993) 74.

[22] M.P. Gelfand and M.E. Fisher, Physica A 166 (1990) 1.

[23] M. Hasenbusch, M. Marcu and K. Pinn,

Nucl. Phys. B (Proc. Suppl.) 26 (1992) 598, and in preparation.

[24] M. Hasenbusch and S. Meyer, Phys. Rev. Lett. 66 (1991) 530.

[25] M. Creutz, Phys. Rev. Lett. 50 (1983) 1411.

[26] M. Creutz, G. Bhanot and H. Neuberger, Nucl. Phys. B235 [FS11] (1984) 417.

[27] M. Creutz, K.J.M. Moriarty and M. O'Brien, Comput. Phys. Comm. 42 (1986) 191. 
[28] K. Rummukainen, Nucl. Phys. B 390 (1993) 621.

[29] G. Bhanot, M. Creutz and J. Lacki, Phys. Rev. Lett. 69 (1992) 1841.

[30] C. Vohwinkel, Phys. Lett. B 301 (1993) 208, and private communication.

[31] H. Arisue and T. Fujiwara, Nucl. Phys. B 285 [FS 19] (1987) 253;

H. Arisue and T. Fujiwara, preprint RIFP-588, 1985, unpublished.

[32] M. Hasenbusch, K. Pinn and K. Rummukainen, in preparation

[33] M.E. Fisher and H. Au-Yang, J. Phys. A 12 (1979) 1677.

[34] M.E. Fisher, private communication.

[35] M.E. Fisher and H. Wen, Phys. Rev. Lett. 68 (1992) 3654.

[36] K.K. Mon, Phys. Rev. Lett. 60 (1988) 2749.

[37] J. Tobochnik and G.V. Chester, Phys. Rev. B 20 (1979) 3761.

[38] R. Gupta, J. Delapp, G.G. Batrouni and G. Fox,

Phys. Rev. Lett. 61 (1988) 1996.

[39] U. Wolff, Nucl. Phys. B 322 (1989) 759.

[40] R.H. Swendsen, Phys. Rev. B 15 (1977) 5421.

[41] R. Baxter, Exactly Solved Models in Statistical Mechanics, Academic 1982.

[42] S. Wansleben, J.G. Zabolitzky and C. Kalle, J. Stat. Phys. 37 (1984) 283.

[43] M. Kikuchi and Y. Okabe, Phys. Rev. B 35 (1987) 5382.

[44] N. Ito and Y. Kanada, Proceedings of Supercomputing '90, IEEE Computer Society Press, 1990, Los Alamitos, p. 753.

[45] N. Ito, private communication.

\section{List of Tables}

$1 \quad$ Algorithm performance . . . . . . . . . . . . . . . . 16

$2 \quad$ Performance of algorithms of other authors . . . . . . . . . . . . . . . 16

3 Ising interface energy at $\beta=0.4500$. . . . . . . . . . . . . . . . 16

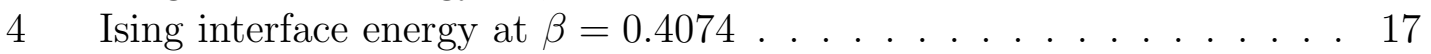

5 Ising interface energy at $\beta=0.3500$. . . . . . . . . . . . . . . . . 17

$6 \quad$ Ising interface energy for $\beta=0.35-0.60$. . . . . . . . . . . . . . . . 18

7 Monte Carlo results for the Ising interface tension . . . . . . . . . . . 19

$8 \quad$ The quantities $\sigma(L) \ldots \ldots \ldots \ldots \ldots \ldots$

$9 \quad$ Low temperature series coefficients $a_{n} \ldots \ldots$. . . . . . . . . . . . . 20

10 Inhomogeneous Differential Approximants . . . . . . . . . . . . . . . 21

11 Monte Carlo results for the energy of the ASOS model . . . . . . . . 21 


\section{Figure Captions}

Fig. 1: Ising surface energy at $\beta=0.5000$ from Padé, truncated series and Monte Carlo. Truncated series estimates are plotted with a ' + ' and connected with a broken line. The close to diagonal Padé estimates are given with diamonds. The data with error bars present our Monte Carlo results for the different lattice sizes. The $L=64$ estimate is also given by two vertical lines.

Fig. 2: Ising surface energy at $\beta=0.4500$ from Padé, truncated series and Monte Carlo. The symbols are the same as in fig. 1

Fig. 3: Ising surface energy at $\beta=0.4074$ from Padé, truncated series and Monte Carlo. The symbols are the same as in fig. 1

Fig. 4: Ising surface tension at $\beta=0.4500$ from Padé, truncated series and Monte Carlo. The symbols are the same as in fig. 1

Fig. 5: Ising surface tension at $u=0.2(\beta=0.4204)$ from Padé, truncated series and Monte Carlo. For comparison we also quote here the Shaw and Fisher estimate (based on the ninth order series evaluation). The symbols are the same as in fig. 1

Fig. 6: The surface tension obtained by Monte Carlo simulation (dotted line) is plotted as a function of the inverse temperature $\beta$. The error bars are smaller than the line width. For comparison we give the interpolation result of Fisher et al. [34, 4, 35] (solid line). The roughening transition at $\beta_{r}=0.4047(3)$ is indicated by a vertical dashed line.

Fig. 7: The derivative of the surface energy with respect to $\beta$ is plotted as a function of the inverse temperature $\beta$. The dotted lines gives the derivative obtained from finite differences of the surface energy for $L=8,16,32$, and 64, computed by Monte Carlo. The height of the peak grows with increasing $L$. For comparison we give the result from the series truncated at order 12 (Series12) and truncated at order 17 (Series17). The roughening transition at $\beta_{r}$ is indicated by a vertical dashed line.

Fig. 8: ASOS energy at $\beta=0.85$ from Padé, truncated series and MC. The symbols are the same as in fig. 1 .

Fig. 9: ASOS energy at $\beta=0.81$ from Padé, truncated series and MC. The symbols are the same as in fig. 1 . 


\begin{tabular}{|c|c|r|}
\hline machine & algorithm & mspins/sec \\
\hline SUN ELC & $\mathrm{mc}$ & 2.3 \\
SUN ELC & $\mathrm{c}$ & 1.1 \\
SPARC 10 & $\mathrm{mc}$ & 7.1 \\
SPARC 10 & $\mathrm{c}$ & 3.2 \\
HP 9000/735 & $\mathrm{mc}$ & 28.0 \\
HP 9000/735 & $\mathrm{c}$ & 14.7 \\
\hline
\end{tabular}

Table 1: Performance of the multi-spin program on various workstations, given in units of $10^{6}$ spin updates per second. 'mc' means micro-canonical run, 'c' with canonical update of the demons

\begin{tabular}{|c|c|c|r|}
\hline author(s) & year & machine & mspins/sec \\
\hline Wansleben et al. [42] & 1984 & CYBER 205 (two pipes) & 21.2 \\
Creutz et al. [27] & 1986 & CYBER 205 (two pipes) & 117.0 \\
Kikuchi and Okabe [43] & 1987 & NEC SX-2 & 251.0 \\
Ito and Kanada 444] & 1990 & HITAC S820/80 & 1960.0 \\
Ito [45] & 1991 & VP2600/10 & 2190.0 \\
\hline
\end{tabular}

Table 2: Performance of the algorithms of other authors on supercomputers for comparison with table 1

\begin{tabular}{|c|l|l|l|c|c|}
\hline$t$ & \multicolumn{1}{|c|}{$L=4$} & \multicolumn{1}{c|}{$L=8$} & \multicolumn{1}{c|}{$L=16$} & \multicolumn{1}{c|}{$L=32$} & \multicolumn{1}{c|}{$L=64$} \\
\hline 2 & $2.67433(63)$ & $2.78942(42)$ & $2.83759(30)$ & $2.85006(21)$ & $2.85225(12)$ \\
3 & $2.66940(57)$ & $2.75544(34)$ & $2.77860(21)$ & $2.78114(11)$ & $2.781092(56)$ \\
4 & $2.66799(63)$ & $2.75238(38)$ & $2.77445(23)$ & $2.77717(13)$ & $2.776936(68)$ \\
5 & $2.66775(57)$ & $2.75246(35)$ & $2.77440(20)$ & $2.77677(11)$ & $2.776790(64)$ \\
9 & $2.6700(16)$ & $2.7517(9)$ & $2.7746(5)$ & & \\
10 & $2.66645(75)$ & $2.75223(42)$ & $2.77442(25)$ & $2.77683(15)$ & $2.776835(83)$ \\
17 & $2.6688(19)$ & $2.7541(11)$ & $2.774(1)$ & & \\
\hline
\end{tabular}

Table 3: $\quad$ Ising interface energy at $\beta=0.4500$ 


\begin{tabular}{|c|l|l|l|l|l|}
\hline$t$ & \multicolumn{1}{|c|}{$L=4$} & \multicolumn{1}{|c|}{$L=8$} & \multicolumn{1}{c|}{$L=16$} & \multicolumn{1}{c|}{$L=32$} & \multicolumn{1}{|c|}{$L=64$} \\
\hline 2 & $2.9542(7)$ & $3.2408(5)$ & $3.42063(27)$ & $3.48668(12)$ & $3.492507(59)$ \\
3 & $2.9479(7)$ & $3.14030(37)$ & $3.21718(22)$ & $3.24322(12)$ & $3.25120(7)$ \\
4 & $2.9419(8)$ & $3.1244(4)$ & $3.19578(24)$ & $3.21901(14)$ & $3.226227(83)$ \\
5 & $2.9414(7)$ & $3.12342(37)$ & $3.19428(22)$ & $3.21745(12)$ & $3.224334(62)$ \\
6 & $2.9427(8)$ & $3.1230(5)$ & $3.19410(24)$ & $3.21735(14)$ & $3.224389(83)$ \\
9 & $2.9384(20)$ & $3.1235(11)$ & $3.1936(6)$ & $3.21723(30)$ & \\
10 & & & & & $3.224219(88)$ \\
11 & $2.9520(10)$ & $3.1237(5)$ & $3.19443(28)$ & $3.21756(16)$ & \\
17 & $2.9431(25)$ & $3.1247(14)$ & $3.1941(7)$ & & \\
33 & & & $3.1946(9)$ & & \\
\hline
\end{tabular}

Table 4: Ising interface energy at $\beta=0.4074$. We made an additional run with $L=128$ and $t=11$. The result for the surface energy is $3.226375(45)$

\begin{tabular}{|c|l|l|l|l|l|}
\hline$t$ & \multicolumn{1}{|c|}{$L=4$} & \multicolumn{1}{|c|}{$L=8$} & \multicolumn{1}{|c|}{$L=16$} & \multicolumn{1}{c|}{$L=32$} & \multicolumn{1}{|c|}{$L=64$} \\
\hline 2 & $3.3173(7)$ & $3.69719(30)$ & $3.77344(14)$ & $3.77574(7)$ & $3.775673(34)$ \\
3 & $3.3684(8)$ & $3.70942(48)$ & $3.85405(31)$ & $3.92648(21)$ & $3.95577(11)$ \\
4 & $3.3479(9)$ & $3.62530(50)$ & $3.70230(29)$ & $3.72218(16)$ & $3.726973(76)$ \\
5 & $3.3432(9)$ & $3.61092(48)$ & $3.68357(28)$ & $3.70146(14)$ & $3.705558(61)$ \\
6 & $3.3437(11)$ & $3.60982(55)$ & $3.68145(28)$ & $3.69886(16)$ & $3.703239(76)$ \\
7 & $3.3446(13)$ & $3.61025(63)$ & $3.68106(32)$ & $3.69870(17)$ & $3.702884(85)$ \\
9 & $3.3431(28)$ & $3.6094(15)$ & $3.6816(8)$ & $3.69854(39)$ & \\
13 & $3.3440(16)$ & $3.61122(78)$ & $3.68080(39)$ & $3.69851(20)$ & $3.70282(10)$ \\
17 & $3.3411(38)$ & $3.6102(19)$ & $3.6836(10)$ & & \\
\hline
\end{tabular}

Table 5: Ising interface energy at $\beta=0.3500$ 


\begin{tabular}{|c|c|c|c|c|c|c|}
\hline$\beta$ & $L=8$ & $L=16$ & $L=32$ & $L=64$ & $S_{17}$ & $S_{12}$ \\
\hline 0.350 & $3.61122(78)$ & $3.68080(39)$ & $3.69851(20)$ & $\overline{3.70282(11)}$ & 4.59933 & 5.77307 \\
\hline 0.360 & $3.53379(70)$ & $3.60450(36)$ & $3.62157(19)$ & $3.62574(9)$ & 4.47093 & 5.09037 \\
\hline 0.370 & $3.45279(67)$ & $3.52385(35)$ & $3.54189(18)$ & $3.54621(9)$ & 4.23424 & 4.56179 \\
\hline 0.380 & $3.36878(64)$ & $3.44095(33)$ & $3.46002(16)$ & $3.46462(8)$ & 3.97351 & 4.14707 \\
\hline 0.390 & $3.28038(59)$ & $3.35522(34)$ & $3.37550(16)$ & $3.38049(7)$ & 3.72531 & 3.81746 \\
\hline 0.395 & & & $3.33163(16)$ & & 3.61065 & 3.67785 \\
\hline 0.400 & $3.19154(55)$ & $3.26458(30)$ & $3.28669(16)$ & $3.29260(8)$ & 3.50320 & 3.55224 \\
\hline 0.405 & & & $3.24002(15)$ & & 3.40309 & 3.43889 \\
\hline 0.410 & $3.09971(53)$ & $3.16889(29)$ & $3.19217(16)$ & $3.19938(9)$ & 3.31017 & 3.33632 \\
\hline 0.415 & & $3.11972(29)$ & $3.14166(16)$ & & 3.22415 & 3.24326 \\
\hline 0.420 & $3.00972(50)$ & $3.06903(29)$ & $3.09000(17)$ & $3.09669(11)$ & 3.14463 & 3.15860 \\
\hline 0.425 & & $3.01806(28)$ & $3.03614(18)$ & & 3.07117 & 3.08140 \\
\hline 0.430 & $2.91929(48)$ & $2.96759(27)$ & $2.98205(18)$ & $2.98512(11)$ & 3.00334 & 3.01083 \\
\hline 0.435 & & $2.91664(28)$ & $2.92813(18)$ & & 2.94069 & 2.94618 \\
\hline 0.440 & $2.83342(47)$ & $2.86806(27)$ & $2.87502(16)$ & $2.87575(9)$ & 2.88278 & 2.88681 \\
\hline 0.445 & & $2.82025(26)$ & $2.82450(16)$ & & 2.82923 & 2.83218 \\
\hline 0.450 & $2.75223(42)$ & $2.77442(25)$ & $2.77682(15)$ & $2.77683(8)$ & 2.77965 & 2.78182 \\
\hline 0.455 & & $2.73093(23)$ & $2.73180(14)$ & & 2.73370 & 2.73529 \\
\hline 0.460 & $2.67688(41)$ & $2.68951(23)$ & $2.68978(14)$ & $2.68991(7)$ & 2.69107 & 2.69224 \\
\hline 0.465 & & $2.65063(21)$ & $2.65065(13)$ & & 2.65148 & 2.65234 \\
\hline 0.470 & $2.60753(38)$ & $2.61440(20)$ & $2.61426(13)$ & $2.61408(6)$ & 2.61465 & 2.61528 \\
\hline 0.475 & & & $2.57979(12)$ & & 2.58036 & 2.58083 \\
\hline 0.480 & $2.54599(36)$ & $2.54823(19)$ & $2.54813(12)$ & $2.54813(6)$ & 2.54840 & 2.54874 \\
\hline 0.485 & & & $2.51866(11)$ & & 2.51856 & 2.51882 \\
\hline 0.490 & $2.48992(32)$ & $2.49041(18)$ & $2.49060(10)$ & $2.49054(5)$ & 2.49068 & 2.49087 \\
\hline 0.500 & $2.44055(30)$ & $2.44052(18)$ & $2.44014(10)$ & $2.44014(5)$ & 2.44018 & 2.44028 \\
\hline 0.510 & $2.39611(28)$ & $2.39613(18)$ & $2.39578(9)$ & & 2.39580 & 2.39586 \\
\hline 0.520 & $2.35725(25)$ & $2.35658(17)$ & $2.35657(8)$ & & 2.35665 & 2.35668 \\
\hline 0.530 & $2.32212(23)$ & $2.32219(16)$ & $2.32208(8)$ & & 2.32198 & 2.32200 \\
\hline 0.540 & $2.29135(23)$ & $2.29123(14)$ & $2.29129(7)$ & & 2.29118 & 2.29119 \\
\hline 0.550 & $2.26399(20)$ & $2.26403(14)$ & $2.26363(7)$ & & 2.26374 & 2.26375 \\
\hline 0.560 & $2.23930(20)$ & $2.23943(13)$ & $2.23904(7)$ & & 2.23922 & 2.23923 \\
\hline 0.570 & $2.21751(19)$ & $2.21728(12)$ & $2.21724(6)$ & & 2.21726 & 2.21726 \\
\hline 0.580 & $2.19748(19)$ & $2.19757(11)$ & & & 2.19755 & 2.19755 \\
\hline 0.590 & $2.17986(17)$ & $2.17989(11)$ & & & 2.17981 & 2.17981 \\
\hline 0.600 & $2.16378(17)$ & $2.16387(10)$ & & & 2.16383 & 2.16383 \\
\hline
\end{tabular}

Table 6: Ising interface energy, Monte Carlo and truncated low temperature series results $\left(S_{i}\right.$ corresponds to truncation at $i^{\text {th }}$ order) 


\begin{tabular}{|c|c|c|c|c|c|c|c|c|}
\hline$\beta$ & $\sigma /(2 \beta)$ & $\mathrm{X}$ & $\bar{\beta}$ & $\sigma /(2 \beta)$ & $\mathrm{X}$ & $\beta$ & $\sigma /(2 \beta)$ & $\mathrm{X}$ \\
\hline \multirow[t]{2}{*}{.225} & $.01629(30)$ & 2.3 & \multirow[t]{2}{*}{.315} & $.575804(38)$ & 7.0 & \multirow[t]{2}{*}{.405} & $.8499891(27)$ & 6.2 \\
\hline & $.01622(25)$ & 2.0 & & $.575824(38)$ & 5.8 & & $.8499979(26)$ & 21.5 \\
\hline \multirow[t]{2}{*}{.230} & $.04929(25)$ & 4.8 & \multirow[t]{2}{*}{.320} & $.597570(31)$ & 6.9 & \multirow[t]{2}{*}{.410} & $.8592883(27)$ & 2.1 \\
\hline & $.04921(20)$ & 3.0 & & $.597585(35)$ & 6.5 & & $.8592906(22)$ & 3.2 \\
\hline \multirow[t]{2}{*}{.235} & $.08565(18)$ & 1.9 & \multirow[t]{2}{*}{.325} & $.618399(28)$ & 5.6 & \multirow[t]{2}{*}{.415} & $.8680694(26)$ & 2.0 \\
\hline & $.08562(20)$ & 1.5 & & $.618420(25)$ & 8.1 & & $.8680646(22)$ & 7.8 \\
\hline \multirow[t]{2}{*}{.240} & $.12262(18)$ & 1.1 & \multirow[t]{2}{*}{.330} & $.638353(26)$ & 7.4 & \multirow[t]{2}{*}{.420} & $.8763354(26)$ & 10.0 \\
\hline & $.12263(16)$ & 1.1 & & $.638390(25)$ & 11.3 & & $.8763233(21)$ & 49.7 \\
\hline \multirow[t]{2}{*}{.245} & $.15971(15)$ & 1.4 & \multirow[t]{2}{*}{.335} & $.657448(21)$ & 4.9 & \multirow[t]{2}{*}{.425} & $.8840889(24)$ & 19.2 \\
\hline & $.15972(14)$ & 1.2 & & $.657500(16)$ & 15.1 & & $.8840714(19)$ & 117.0 \\
\hline \multirow[t]{2}{*}{.250} & $.19623(14)$ & 1.2 & \multirow[t]{2}{*}{.340} & $.675699(13)$ & 2.8 & \multirow[t]{2}{*}{.430} & $.8913357(25)$ & 24.3 \\
\hline & $.19627(12)$ & 1.2 & & $.675751(12)$ & 23.6 & & $.8913154(18)$ & 178.7 \\
\hline \multirow[t]{2}{*}{.255} & $.23200(12)$ & 1.4 & \multirow[t]{2}{*}{.345} & $.6931576(53)$ & 3.7 & \multirow[t]{2}{*}{.435} & $.8980930(21)$ & 13.9 \\
\hline & $.23202(12)$ & 1.5 & & $.6931802(49)$ & 39.6 & & $.8980731(16)$ & 195.0 \\
\hline \multirow[t]{2}{*}{.260} & $.266799(93)$ & 1.7 & \multirow[t]{2}{*}{.350} & $.7098453(66)$ & 1.0 & \multirow[t]{2}{*}{.440} & $.9043836(18)$ & 5.2 \\
\hline & .266831(94) & 1.8 & & $.7098713(62)$ & 34.5 & & $.9043665(16)$ & 175.3 \\
\hline \multirow[t]{2}{*}{.265} & $.300562(97)$ & 1.5 & \multirow[t]{2}{*}{.355} & $.7258015(44)$ & 2.5 & .445 & $.9102364(18)$ & 2.0 \\
\hline & $.300592(92)$ & 2.2 & & $.7258219(36)$ & 43.5 & & $.9102225(14)$ & 137.8 \\
\hline .270 & $.333177(90)$ & 2.4 & .360 & $.7410463(39)$ & 5.6 & .450 & $.9156833(14)$ & .9 \\
\hline & $.333196(80)$ & 2.6 & & $.7410681(34)$ & 51.9 & & $.9156726(12)$ & 97.3 \\
\hline .275 & $.364607(73)$ & 2.9 & .365 & $.7556038(42)$ & 5.1 & .455 & $.9207540(12)$ & 1.6 \\
\hline & $.364639(82)$ & 2.7 & & $.7556250(33)$ & 59.9 & & $.9207468(12)$ & 61.3 \\
\hline .280 & $.394894(71)$ & 1.7 & .370 & $.7694993(37)$ & 4.7 & .460 & $.9254779(11)$ & 1.6 \\
\hline & $.394923(66)$ & 2.3 & & $.7695206(34)$ & 64.3 & & $.9254730(10)$ & 36.2 \\
\hline .285 & $.424033(66)$ & 1.8 & .375 & $.7827565(36)$ & 6.7 & .465 & $.9298820(12)$ & 1.3 \\
\hline & $.424051(60)$ & 2.3 & & $.7827780(30)$ & 68.5 & & $.9298786(10)$ & 20.3 \\
\hline .290 & $.451998(61)$ & 1.8 & .380 & $.7953967(37)$ & 8.4 & .470 & $.9339903(10)$ & 2.1 \\
\hline & $.452023(60)$ & 2.6 & & $.7954174(32)$ & 70.4 & & $.9339883(09)$ & 9.2 \\
\hline .295 & $.478878(63)$ & 2.2 & .385 & $.8074397(33)$ & 9.2 & .475 & $.9378262(08)$ & 1.3 \\
\hline & $.478887(55)$ & 2.6 & & $.8074602(31)$ & 69.4 & & $.9378251(07)$ & 3.9 \\
\hline .300 & $.504651(53)$ & 2.8 & .390 & $.8189052(34)$ & 10.6 & .480 & $.9414105(06)$ & 1.0 \\
\hline & $.504664(52)$ & 2.9 & & $.8189246(26)$ & 66.9 & & $.9414100(06)$ & 2.5 \\
\hline .305 & $.529376(47)$ & 3.8 & .395 & $.8298090(32)$ & 10.5 & .485 & $.9447624(04)$ & 1.6 \\
\hline & $.529393(46)$ & 3.5 & & $.8298263(28)$ & 59.4 & & $.9447621(03)$ & 1.8 \\
\hline .310 & $.553076(44)$ & 5.2 & .400 & $.8401660(29)$ & 10.1 & .490 & $.9478999(04)$ & 1.8 \\
\hline & $.553089(42)$ & 4.3 & & $.8401801(25)$ & 43.6 & & $.9478997(03)$ & 1.1 \\
\hline
\end{tabular}

Table 7: Monte Carlo results for the interface tension. We always quote two numbers. For the upper number the $L=16,32,64$ data were included. For lower number also the $L=8$ data were included. X denotes $\chi^{2}$ per degree of freedom for the fit of the free energies with $C_{s}+\sigma L^{2}$ 


\begin{tabular}{|rl|rl|}
\hline \multicolumn{4}{|c|}{$\beta=0.402359(u=0.2)$} \\
\hline$L$ & $\sigma(L) /(2 \beta)$ & pair & $\sigma /(2 \beta)$ \\
\hline 8 & $0.849503(17)$ & $8-16$ & 0.844959 \\
16 & $0.8460946(87)$ & $16-32$ & 0.844882 \\
32 & $0.8451848(42)$ & $32-64$ & 0.844858 \\
64 & $0.8449398(30)$ & & \\
\hline \hline \multicolumn{4}{|c|}{$\beta=0.45$} \\
\hline$L$ & $\sigma(L) /(2 \beta)$ & pair & $\sigma /(2 \beta)$ \\
\hline 8 & $0.916039(10)$ & $8-16$ & 0.915567 \\
16 & $0.9156853(56)$ & $16-32$ & 0.915683 \\
32 & $0.9156838(24)$ & $32-64$ & 0.915683 \\
64 & $0.9156833(14)$ & & \\
\hline
\end{tabular}

Table 8: $\quad$ Surface tension $\sigma(L) /(2 \beta)$ together with estimates for the same quantity obtained from surface free energies for pairs of $L$-values

\begin{tabular}{|r|l|l|}
\hline$n$ & \multicolumn{1}{|c|}{$a_{n}^{\text {Ising }}$} & \multicolumn{1}{|c|}{$a_{n}^{\text {ASOS }}$} \\
\hline 2 & -2 & -2 \\
3 & -2 & -4 \\
4 & -10 & -10 \\
5 & -16 & -24 \\
6 & $-242 / 3$ & $-194 / 3$ \\
7 & -150 & -172 \\
8 & -734 & -452 \\
9 & $-4334 / 3$ & $-3184 / 3$ \\
10 & $-32122 / 5$ & $-8862 / 5$ \\
11 & -10224 & +1712 \\
12 & $-106348 / 3$ & $+116804 / 3$ \\
13 & +53076 & \\
14 & $+3491304 / 7$ & \\
15 & $+74013814 / 15$ & \\
16 & +27330236 & \\
17 & +160071418 & \\
\hline
\end{tabular}

Table 9: Coefficients of the low temperature series for the 3D Ising and ASOS interface tension 


\begin{tabular}{|c|c|c|c|c|c|c|c|}
\hline \multicolumn{2}{|c|}{ order 17} & \multicolumn{2}{|c|}{ order 15} & \multicolumn{2}{|c|}{ order 12} & \multicolumn{2}{|c|}{ order 9} \\
\hline$[9 / 5,1]$ & - & {$[7 / 5,1]$} & 0.84919 & {$[6 / 3,1]$} & 0.84135 & {$[4 / 2,1]$} & 0.84073 \\
\hline$[8 / 6,1]$ & 0.84508 & {$[6 / 6,1]$} & 0.84621 & {$[5 / 4,1]$} & 0.84364 & {$[3 / 3,1]$} & 0.84069 \\
\hline$[7 / 7,1]$ & - & {$[5 / 7,1]$} & 0.84618 & {$[4 / 5,1]$} & 0.84126 & {$[2 / 4,1]$} & - \\
\hline$[6 / 8,1]$ & - & & & {$[3 / 6,1]$} & 0.84125 & & \\
\hline$[9 / 4,2]$ & 0.84606 & {$[7 / 4,2]$} & - & {$[5 / 3,2]$} & 0.84145 & {$[3 / 2,2]$} & 0.84041 \\
\hline$[8 / 5,2]$ & 0.84566 & {$[6 / 5,2]$} & 0.84480 & {$[4 / 4,2]$} & 0.84143 & {$[2 / 3,2]$} & 0.84029 \\
\hline$[7 / 6,2]$ & 0.84575 & {$[5 / 6,2]$} & 0.84682 & {$[3 / 5,2]$} & 0.84115 & & \\
\hline$[6 / 7,2]$ & 0.84545 & {$[4 / 7,2]$} & 0.84475 & & & & \\
\hline$[5 / 8,2]$ & 0.84592 & & & & & & \\
\hline$[4 / 9,2]$ & 0.84590 & & & & & & \\
\hline$[7 / 5,3]$ & - & {$[6 / 4,3]$} & - & {$[5 / 2,3]$} & 0.84167 & {$[3 / 1,3]$} & 0.84070 \\
\hline$[6 / 6,3]$ & - & {$[5 / 5,3]$} & 0.84579 & {$[4 / 3,3]$} & - & {$[2 / 2,3]$} & 0.84025 \\
\hline$[5 / 7,3]$ & 0.84549 & {$[4 / 6,3]$} & 0.84552 & {$[3 / 4,3]$} & 0.84174 & {$[1 / 3,3]$} & 0.84032 \\
\hline$[4 / 8,3]$ & - & & & {$[2 / 5,3]$} & 0.84206 & & \\
\hline$[7 / 4,4]$ & - & {$[6 / 3,4]$} & - & {$[4 / 2,4]$} & - & & \\
\hline$[6 / 5,4]$ & 0.84555 & {$[5 / 4,4]$} & - & {$[3 / 3,4]$} & 0.84296 & & \\
\hline$[5 / 6,4]$ & 0.84549 & {$[4 / 5,4]$} & - & {$[2 / 4,4]$} & 0.84208 & & \\
\hline$[4 / 7,4]$ & - & {$[3 / 6,4]$} & 0.84490 & & & & \\
\hline$[7 / 3,5]$ & 0.84563 & {$[5 / 3,5]$} & - & & & & \\
\hline$[6 / 4,5]$ & 0.84568 & {$[4 / 4,5]$} & - & & & & \\
\hline$[5 / 5,5]$ & 0.84563 & {$[3 / 5,5]$} & 0.84554 & & & & \\
\hline$[4 / 6,5]$ & - & & & & & & \\
\hline$[3 / 7,5]$ & 0.84566 & & & & & & \\
\hline
\end{tabular}

Table 10: Surface tension $\sigma /(2 \beta)$ at $u=0.2(\beta=0.4024)$ estimated from inhomogeneous differential approximants $[N / M, L]$ for $Q(u)$. The symbol '-' means that the corresponding approximant could not be computed because it did not exist or the numerics was unstable

\begin{tabular}{|c|l|c|l|}
\hline$\beta_{A S O S}$ & \multicolumn{1}{|c|}{$L=64$} & \multicolumn{1}{|c|}{$L=128$} & \multicolumn{1}{|c|}{$L=256$} \\
\hline 0.81 & $0.70741(57)$ & $0.70947(33)$ & $0.70964(18)$ \\
0.82 & $0.67948(63)$ & $0.68052(36)$ & $0.68106(20)$ \\
0.83 & $0.64976(68)$ & $0.65157(40)$ & $0.65182(20)$ \\
0.84 & $0.64976(68)$ & $0.62025(37)$ & $0.62048(20)$ \\
0.85 & $0.5874(8)$ & $0.58837(39)$ & $0.58869(19)$ \\
0.87 & $0.5272(6)$ & $0.52615(35)$ & $0.52643(14)$ \\
0.90 & $0.4420(6)$ & $0.44255(26)$ & $0.44245(14)$ \\
0.95 & $0.3320(6)$ & $0.33177(28)$ & $0.33173(15)$ \\
1.00 & $0.2517(4)$ & $0.25153(23)$ & $0.25128(10)$ \\
1.10 & $0.1496(3)$ & $0.14869(15)$ & $0.14905(7)$ \\
\hline
\end{tabular}

Table 11: Monte Carlo results for the energy of the ASOS model 
Fig. 1: Ising surface energy at beta $=0.5000$ from Pade, truncated series andMC

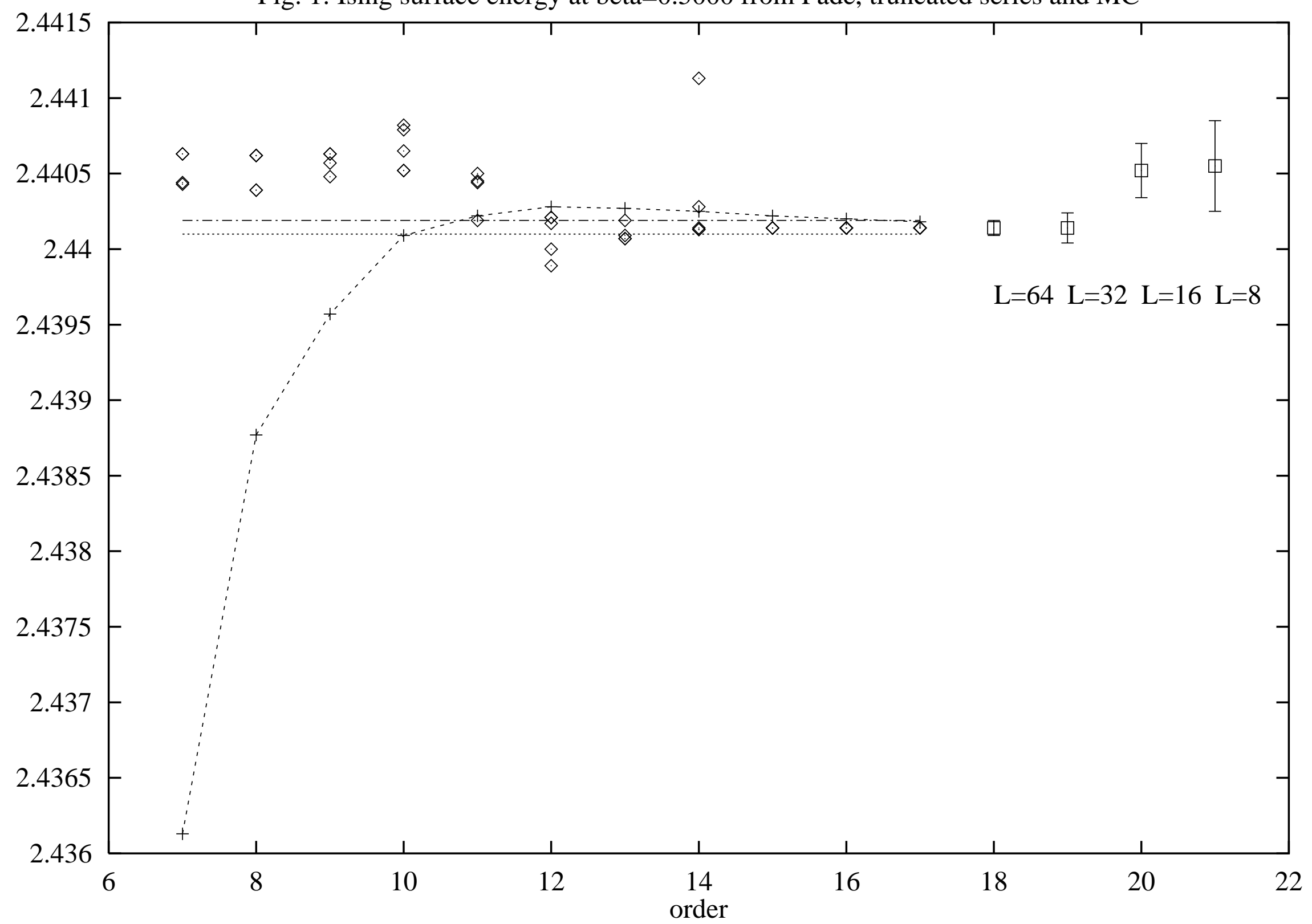


Fig. 2: Ising surface energy at beta $=0.4500$ from Pade, truncated series andMC

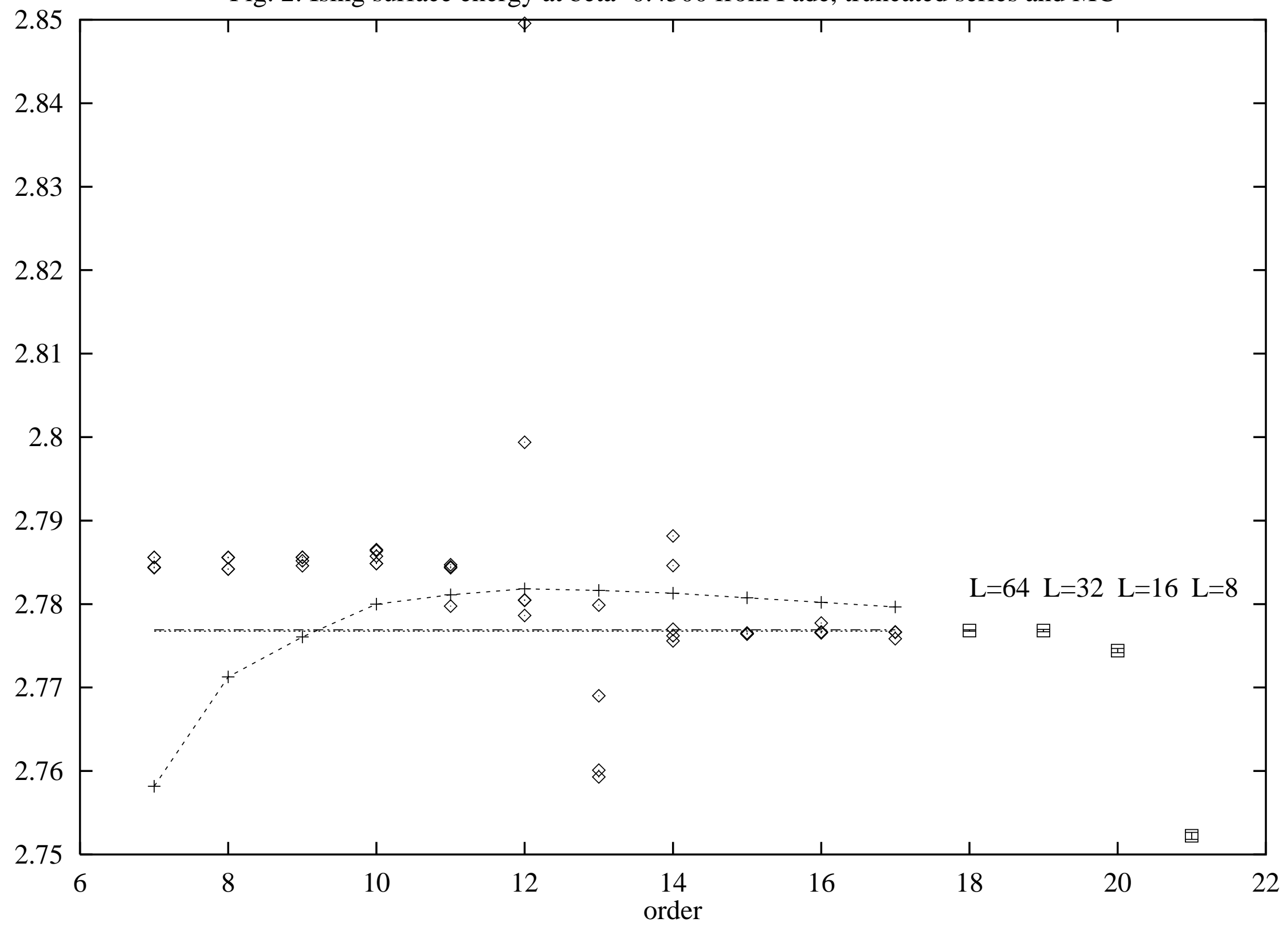


Fig. 3: Ising surface energy at beta $=0.4074$ from Pade, truncated series andMC

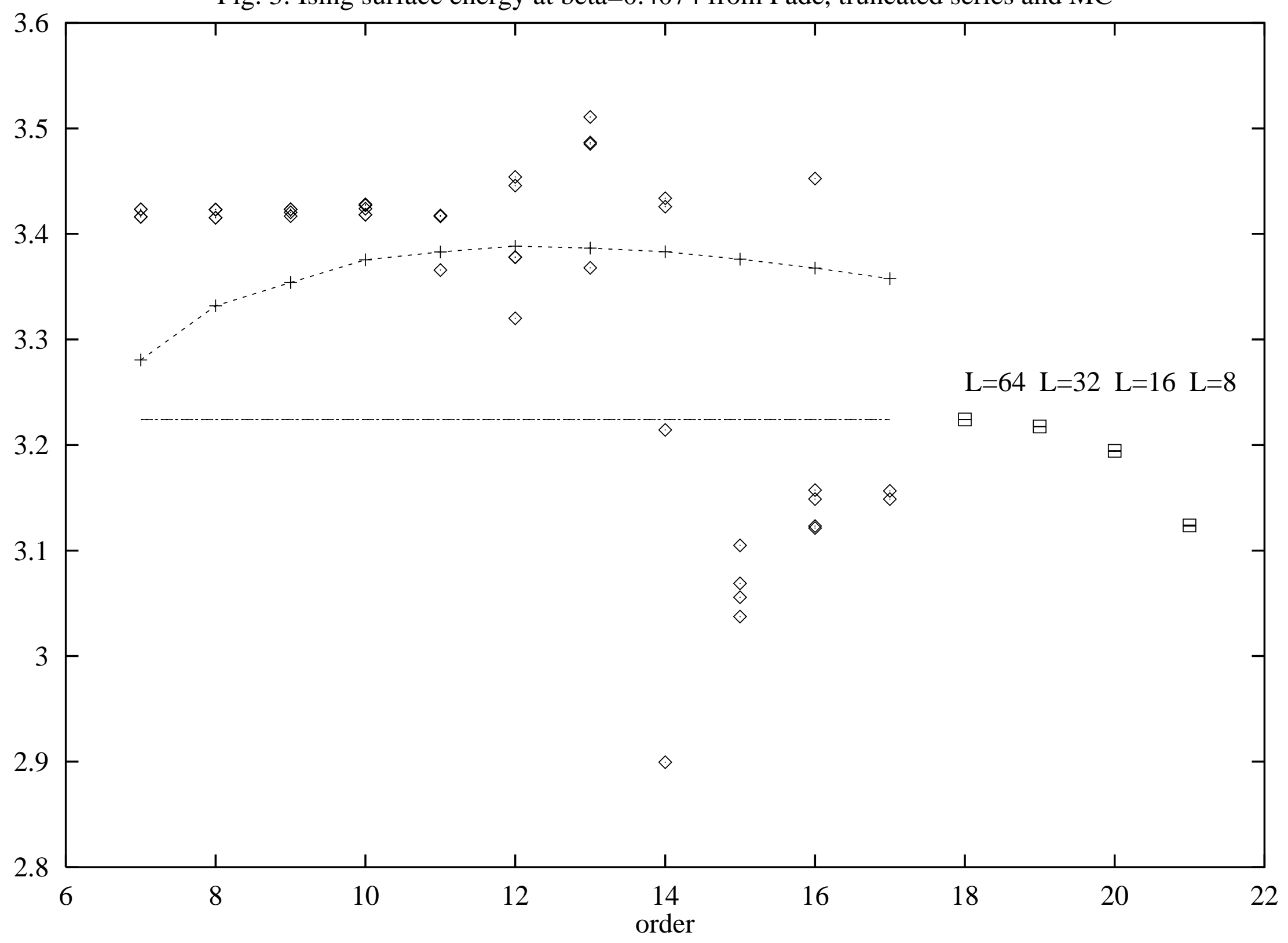


Fig. 4: Ising surface tension at beta $=0.4500$ from Pade, truncated series andMC

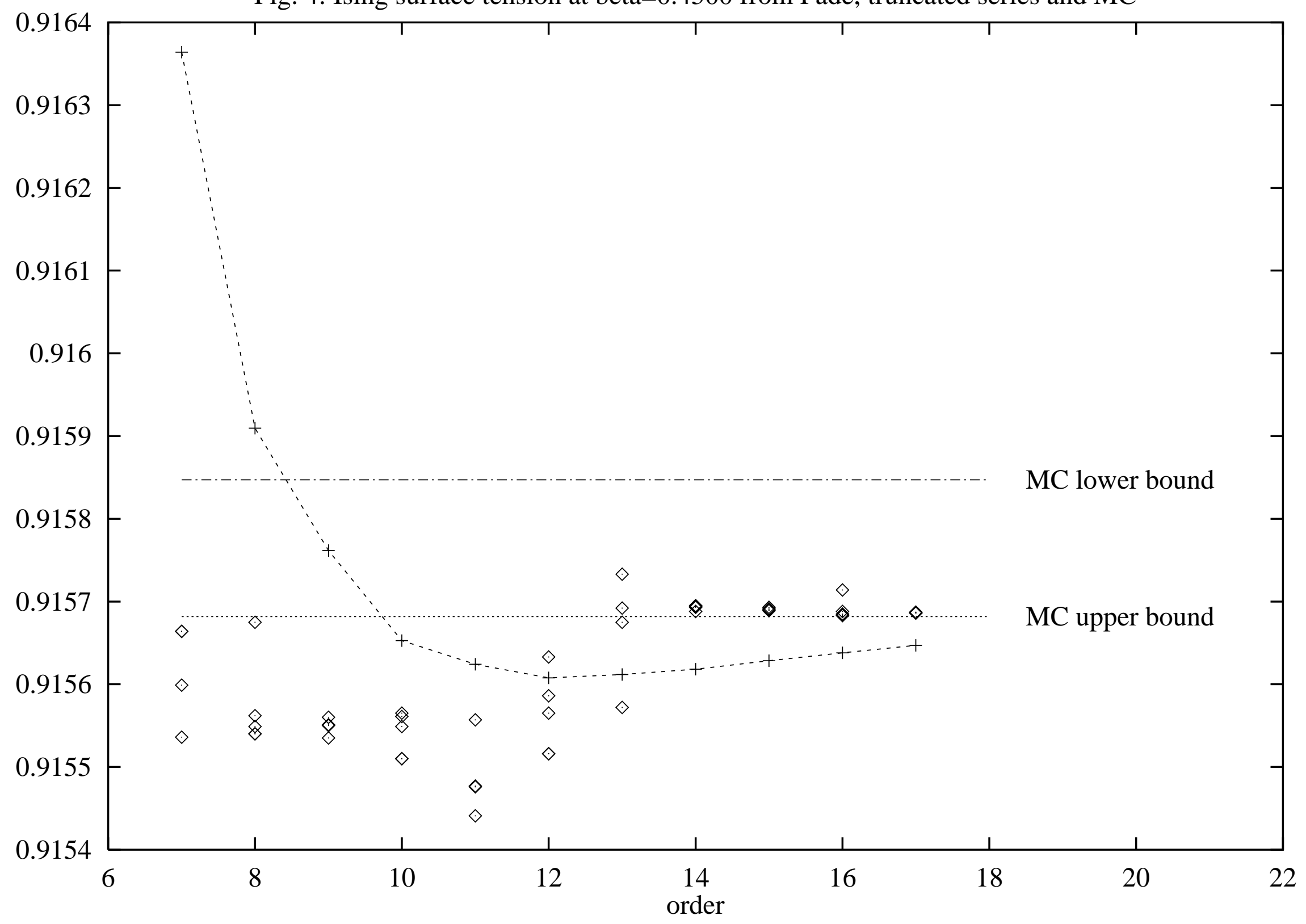




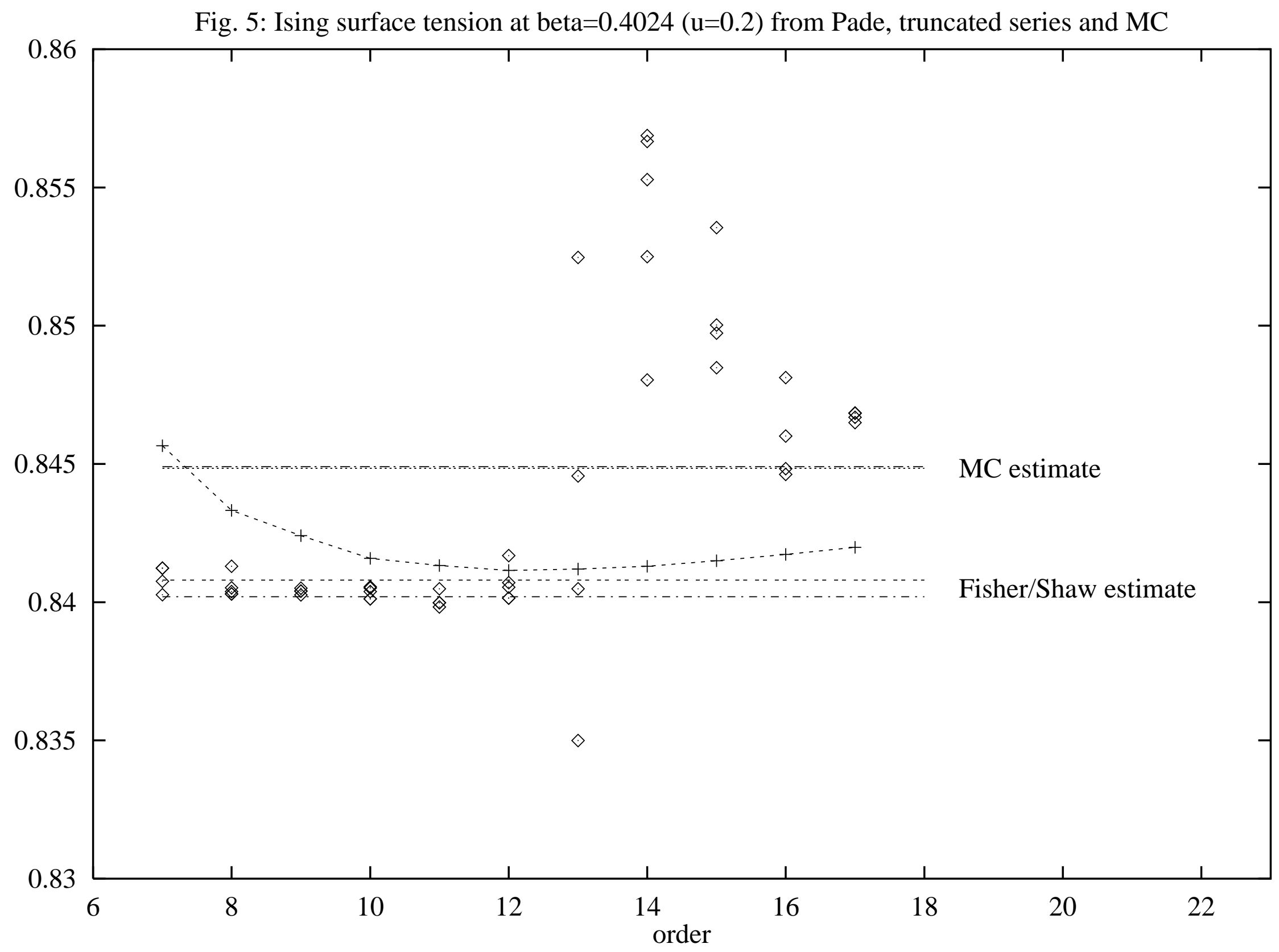


Fig. 6

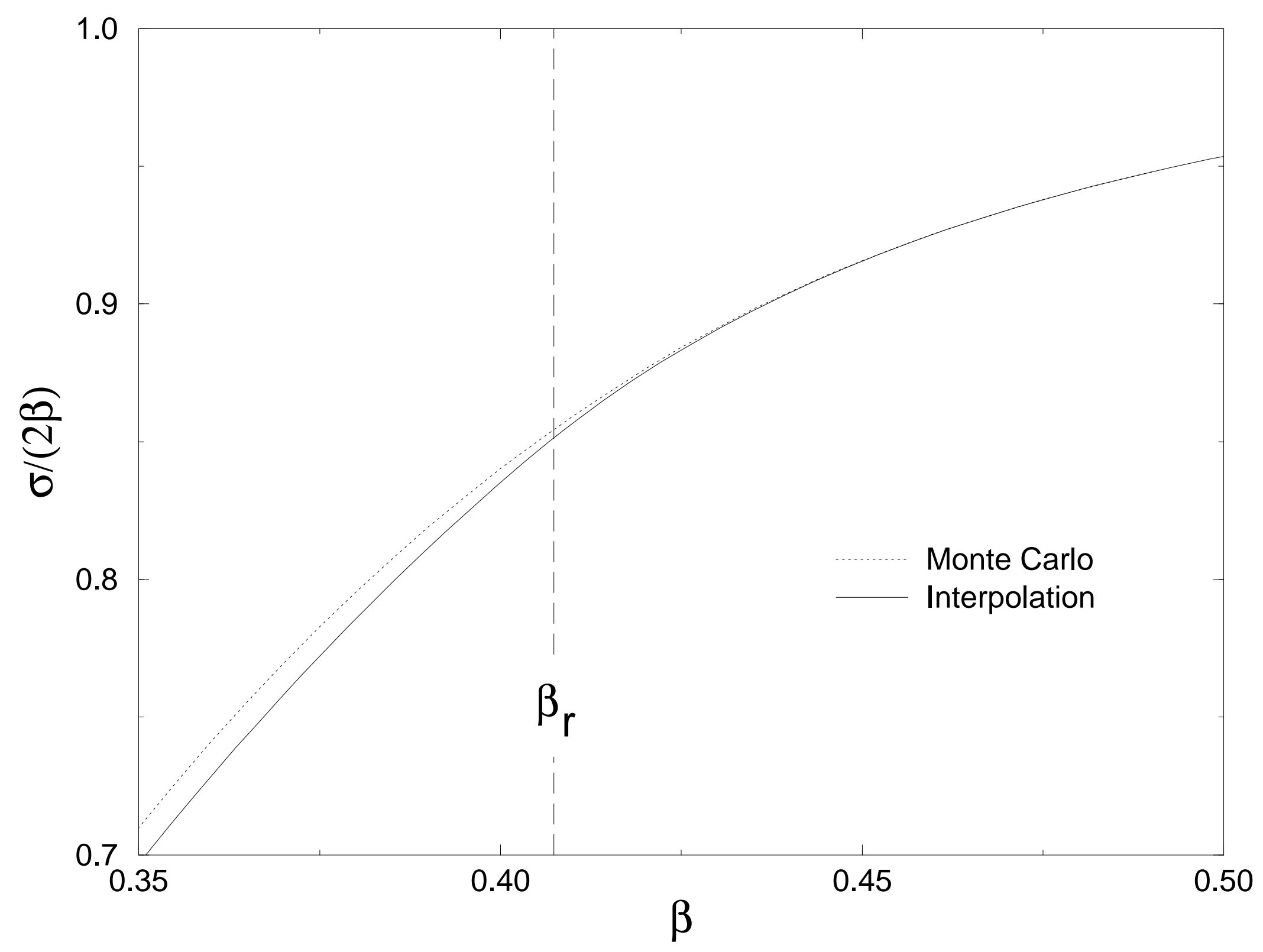


Fig. 7

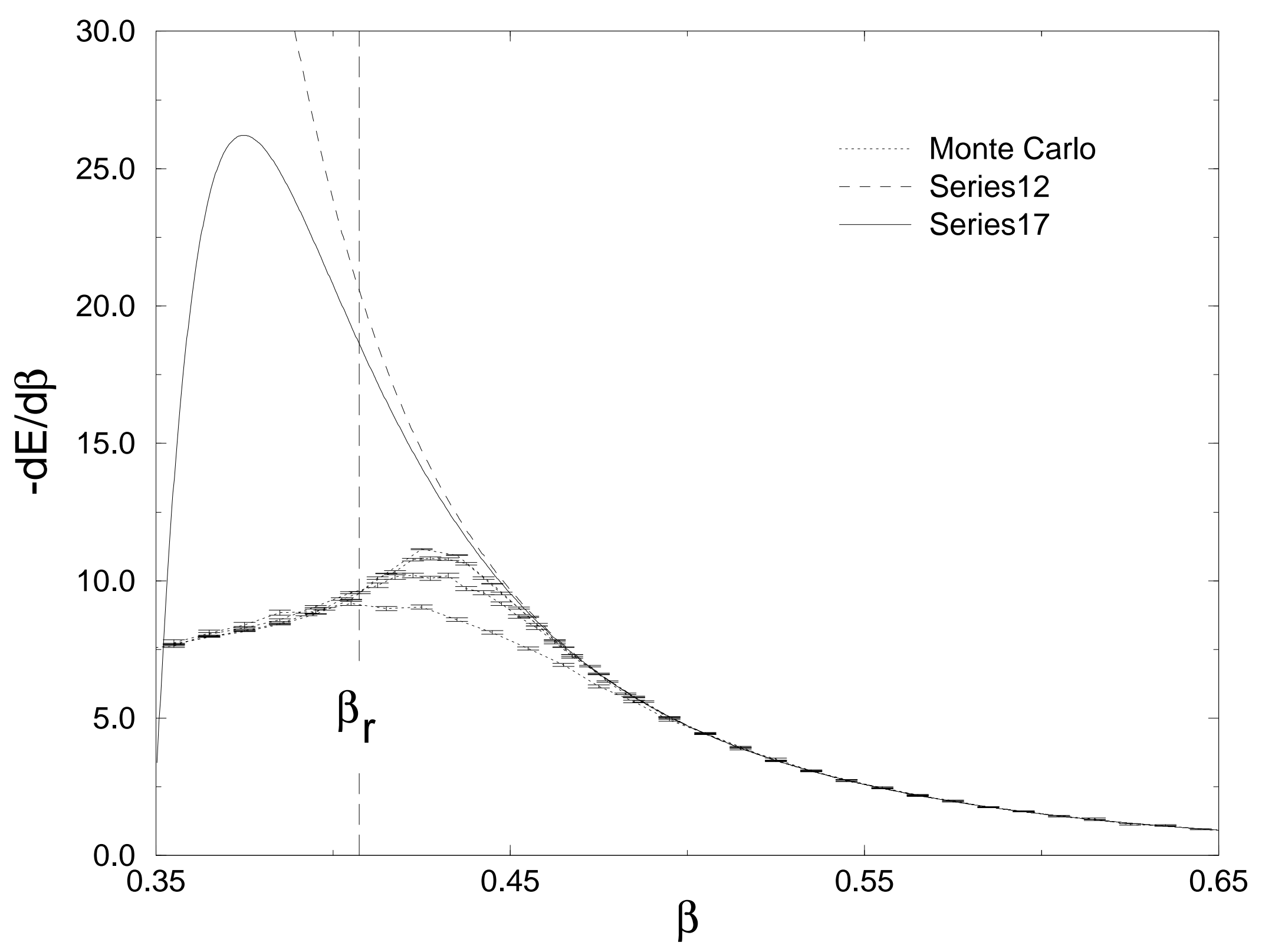


Fig. 8: ASOS energy at beta $=0.85$ from Pade, truncated series and MC

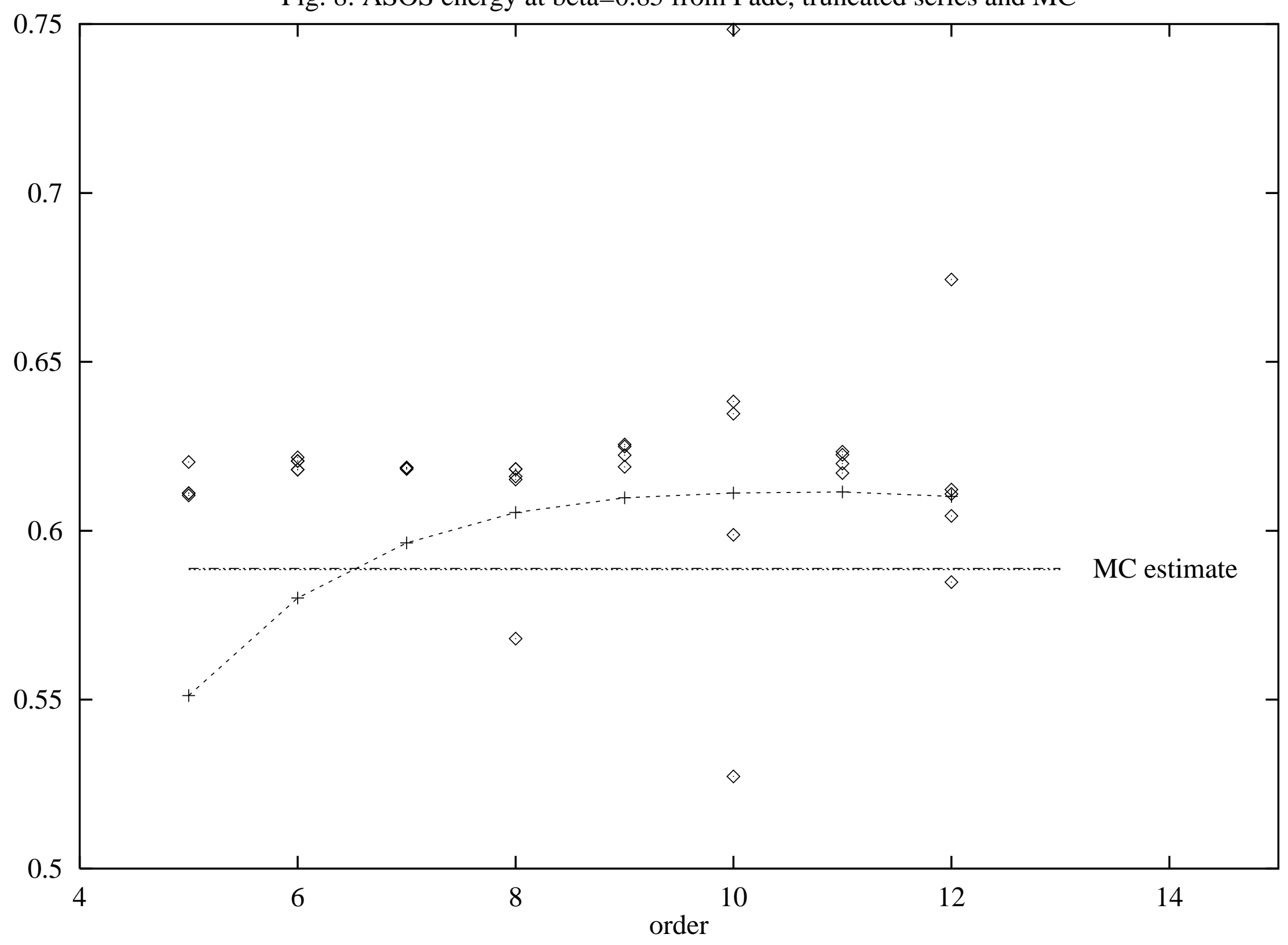


Fig. 9: ASOS energy at beta $=0.81$ from Pade, truncated series and MC

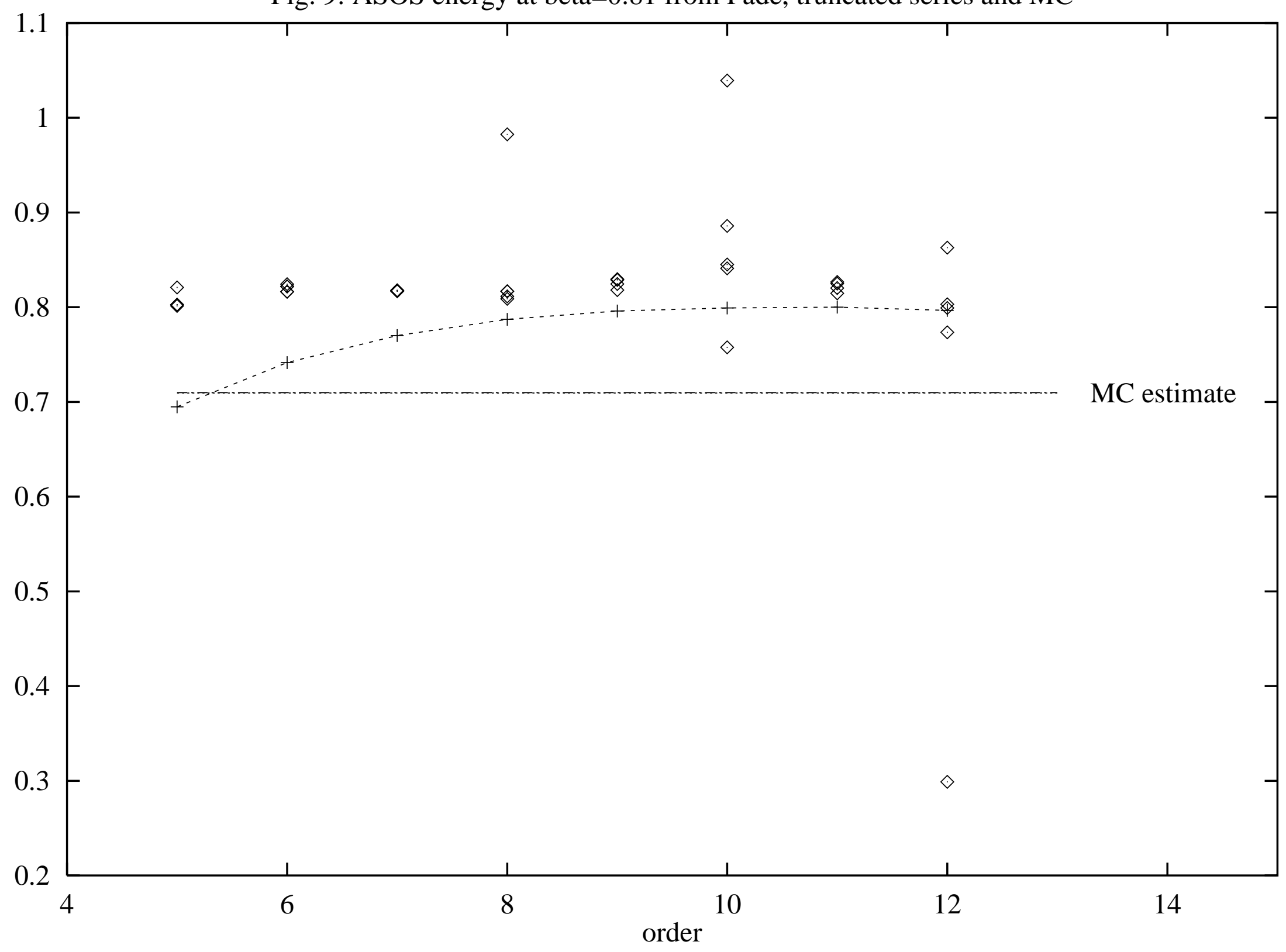

